\title{
Studies on Preparation and Absolute Bioavailability of a Self-Emulsifying System Containing Puerarin
}

\author{
Dong-qin QuAN, ${ }^{* a}$ Gui-xia $\mathrm{Xu},{ }^{a}$ and Xiang-gen $\mathrm{Wu}^{b}$ \\ ${ }^{a}$ Institute of Pharmacology and Toxicology, Academy of Military Medical Sciences; Beijing 100850, China: and \\ ${ }^{b}$ Department of Pharmaceutics, Pharmacy School, Tongji Medical College, Huazhong University of Science and \\ Technology; Wuhan, 430030, China. Received October 12, 2006; accepted January 26, 2007
}

The purpose of the study was to develop an optimum formulation of self-emulsifying drug delivery systems (SEDDS) containing puerarin and to evaluate its absolute bioavailability. Using oleic acid as oil, Tween-80 as surfactant and propylene glycol as cosurfactant, a series of mixtures comprising oleic acid, propylene glycol and Tween 80 were prepared and their self-emulsifying properties were studied. Pseudo-ternary phase diagrams were constructed to identify the efficient self-emulsification region and particle sizes of the resultant emulsions were determined using a laser diffraction sizer. From these studies, an optimized formulation consisting of oil (17.5\%), Tween-80 (34.5\%) and cosurfactant (34.5\%) was selected and its absolute bioavailability in beagle dogs after oral administration was about $\mathbf{2 4 . 8 \%}$. The data suggest the use of SEDDS to provide a potential way of puerarin administered orally.

Key words self-emulsifying system; puerarin; absolute bioavailability

Approximately $40 \%$ of drug candidates have poor water solubility and the oral delivery of such drugs was frequently associated with implications of low bioavailability, high intra- and inter subject variability, and lack of dose proportionality. ${ }^{1)}$ To overcome these problems, various formulation strategies were reported in the literature including the use of surfactants, cyclodextrins, nanoparticles, solid dispersions, micronization, lipids, and permeation enhancers. ${ }^{2)}$ These approaches were successful in selected cases. Nowadays, the interest of self-emulsifying drug delivery systems (SEDDS) to improve the oral bioavailability of poorly watersoluble drugs were well known and documented with various drugs: progesterone, ${ }^{3)}$ halofantrine, ${ }^{4)}$ indomethacin ${ }^{5)}$ and ubiquinone $^{6,7)}$ (coenzyme Q10). SEDDS are isotropic mixture of oils, surfactants, and cosurfactants, which are emulsified in aqueous media under conditions of gentle agitation. ${ }^{8)}$ For drugs with poor water solubility, SEDDS may offer an improvement in both rate and extent of absorption. Selfemulsifying formulations spread readily in the GI tract, and the digestive motility of the stomach and the intestine provide the agitation necessary for self-emulsification. When compared with emulsions, which are sensitive and metastable dispersed forms, SEDDS are physically stable formulations that are easy to manufacture. Thus, for lipophilic drug compounds, SEDDS may offer an improvement in the rate and extent of absorption and more reproducible blood-time profiles. The absorption improvement is through several complex mechanisms such as increased dissolution rate of the drug and solubility in the intestinal fluid. In some cases, as shown recently, excipients incorporated in SEDDS/self-microemulsifying drug delivery systems (SMEDDS) can inhibit both presystemic drug metabolism and intestinal efflux mediated by P-gp resulting in an increased oral absorption of drugs. ${ }^{9)}$

Puerarin known as isoflavone compound is extracted and isolated from kudzuvine root of leguminous plant. It is traditional Chinese medicine used in the treatment of cardiovascular disorders such as arhythmia, hypertension, and myocardial ischemia. Puerarin is practically insoluble in water with low solubility of $0.462 \mathrm{mg} / \mathrm{ml}$ and no oral dosage forms are currently available on the market. In order to administer by oral route of puerarin, the poor aqueous solubility should be improved. Recently, phospholipid puerarin complex studies were reported as a means to improve the oral absorption of puerarin. ${ }^{10)}$ The objectives of the study were to develop an optimum formulation of SEDDS containing puerarin and to evaluate oral absolute bioavailability in dogs. This study may suggest the use of SEDDS to provide a potential way of puerarin administered orally.

\section{Experimental}

Materials and Apparatus Tween 80 and propylene glycol were obtained from Sigma Chem. Co. and soybean oil, oleic acid were purchased from TieLing Pharmaceutical Oil company. MIGLYOL 812N (medium chain triglycerides) was supplied by Gattefossé (France), Cremophor RH 40 (Polyoxyl 40 hydrogenated castor oil) was from BASF (Germany). Beagle dogs were from animal center of Academy of Military Medical Sciences, $13-15 \mathrm{~kg}$, male.

Zetasizer 3000HS was made in Malvern Co., U.K., Hermle Z200A high speed centrifuger was made in German, Hitachi L-7100 HPLC was made in Japan, QL-901 vortex mixer was made in Shanghai, China, UV-160A vis-ultraviolet spectrophotometer was made in SHIMADZU Co., Japan, RC-3B drug dissolution test instrument was made in Tianjin University Factory, China.

Determination of Solubility of Puerarin in Various Oils and Surfactants Excess puerarin was added to various oils or surfactants and mixed with an ultrasonic cleaner at $60^{\circ} \mathrm{C}$ in a water bath for $20 \mathrm{~min}$, then shaken at $37^{\circ} \mathrm{C}$ for $12 \mathrm{~h}$. Samples were filtered through a $0.22 \mu \mathrm{m}$ membrane filter. The filtrate was diluted with methanol and the concentration of puerarin was determined by HPLC method.

Construction of Pseudo-Ternary Phase Diagrams Pseudo-ternary phase diagrams were constructed with the concentrations of oil, surfactant and cosurfactant to form a clear solution in the system. Oleic acid was used as oil phase, Tween 80 was used as surfactant, propylene glycol was used as cosurfactant. The existence of SEDDS fields was identified preliminarily by visual observation described below in 'Preparation of SEDDS of Puerarin'. The efficient self-emulsification region can be drawn according to the particle size distributions of the resultant emulsions which were determined using a laser diffraction sizer.

Preparation of SEDDS of Puerarin SEDDS with varying concentrations of oleic acid (16-32\%), Tween $80(15-55 \%)$, propylene glycol (17$40 \%)$ and puerarin $(100 \mathrm{mg}$ ) were accurately weighed and heated in a water bath at $60^{\circ} \mathrm{C}$ to facilitate the solubilization and the contents were mixed gently with a magnetic stir bar until the mixture solution turned to clear. The formulations were then stored at room temperature. A series of SEDDS in each of six formulations were selected within the self-emulsification region 
Table 1. Solubility of Puerarin in Various Vehicles $(n=3)$

\begin{tabular}{lcccccc}
\hline \hline & \multicolumn{5}{c}{ Solubility of puerarin (mean \pm S.D., mg/g) } \\
\hline Vehicles & Propylene glycol & MCT & SO & OA & EL-40 & Twee-80 \\
Solubility & $572.4 \pm 4.1$ & $32.2 \pm 2.1$ & $59.2 \pm 3.8$ & $124.5 \pm 5.7$ & $103.3 \pm 3.2$ & $168.5 \pm 2.9$ \\
\hline
\end{tabular}

and their self-emulsifying performances were investigated.

Methods to Evaluate Self-Emulsifying Performances Visual Observations: A visual test to assess the self-emulsification properties reported by Craig et al. ${ }^{11)}$ was modified and adopted in the present study. To investigate the quality of resultant emulsion, formulation $(1 \mathrm{~g})$ was introduced into $500 \mathrm{ml}$ of $37^{\circ} \mathrm{C}$ water under a gentle agitation of $50 \mathrm{rpm}$ in a rotating paddle dissolution apparatus. The tendency to emulsify spontaneously were observed. The tendency to form an emulsion was judged as 'good' when droplets spread easily in water and formed a fine milky emulsion, and it was judged 'bad' when there was poor or no emulsion formation with immediate coalescence of oil droplets. On the other hand, $5 \mathrm{ml}$ of the resultant emulsions after agitation $20 \mathrm{~min}$ later were centrifuged at $4000 \mathrm{rpm}$ for $5 \mathrm{~min}$. Coalescence and phase separation were noted. Clearly, this test is qualitative and gives rapid measure of stability of emulsion.

Emulsion Droplet Size Analysis: Formulation (1 g) was diluted with purified water at $37^{\circ} \mathrm{C}$ with a stirring rate of $50 \mathrm{rpm}$ using a rotating paddle dissolution apparatus. The droplet size distribution of the resultant emulsions after $20 \mathrm{~min}$ was determined by a laser diffraction sizer (Zetasizer $3000 \mathrm{HS}$, U.K.), able to measure sizes between 10 and $5000 \mathrm{~nm}$. An analysis of variance procedure (one-way ANOVA) was used to compare the values of the formulations (F1, F2, F3, F4) with regard to the mean droplet sizes.

Bioavailability Studies. Absolute Bioavailability Studies Six beagle dogs were divided into two groups randomly. Each dog received either i.v. puerarin injection $(1 \mathrm{ml}$ of $100 \mathrm{mg} / \mathrm{ml}$ puerarin in $30 \%$ propylene glycol solution given intravenously) or a SEDDS formulation which were filled into soft capsules containing $100 \mathrm{mg}$ puerarin administered by oral [formulation with oleic acid (17.5\%), Tween $80(34.5 \%)$ and propylene glycol (34.5\%)] Approximately $3 \mathrm{ml}$ blood samples were drawn into heparinized tube at 0.25 , $0.5,0.75,1.0,1.25,1.5,2.0,2.5,3.0,4.0,6.0,8.0 \mathrm{~h}$, the blood samples were centrifuged at $3500 \mathrm{rpm}$ for $20 \mathrm{~min}$ and the plasma was separated and kept frozen at $-20{ }^{\circ} \mathrm{C}$. The washout period was one week. The area under the curve $(A U C)$ was calculated by linear trapezoidal rule from zero to the last plasma concentration. Absolute bioavailability can be calculated by ratio of $A U C_{\text {p.o. }} / A U C_{\text {i.v. }}$.

Plasma Sample Analysis: $6 \mathrm{ml}$ absolute methanol was added to $1.0 \mathrm{ml}$ plasma containing hydroxybenzaldehyde (internal standard substance) solution in a $10 \mathrm{ml}$ glass centrifuge tube for assay and mixed well by vortex mixer and centrifuged at $3000 \mathrm{rpm}$ for $15 \mathrm{~min}$. The supernatant phase was separated and the remainder was washed twice by methanol. The combined supernatant was evaporated to dryness at $40{ }^{\circ} \mathrm{C}$ under nitrogen. Residues were dissolved in $200 \mu \mathrm{l}$ methanol and drug content were assayed by HPLC.

HPLC Determination of Puerarin in Plasma: The concentrations of puerarin in the samples were analyzed by slight modification of reported method. ${ }^{12)}$ The mobile phase consisted of methanol- $0.1 \%$ citric acid solution $(25: 75)$, at a flow rate of $1.5 \mathrm{ml} \cdot \mathrm{min}^{-1}$. The column used was Kromacil- $_{18}(4.6 \mathrm{~mm} \times 150 \mathrm{~mm}, 5 \mu \mathrm{m})$. The detection wavelength was $250 \mathrm{~nm}$. The standard curve was constructed by plotting the ratio of peak area of puerarin to that of hydroxybenzaldehyde (internal standard substance) against the known concentrations of puerarin added to blank plasma in the range of $0.0922,0.1844,0.461,0.922,1.844,4.610 \mathrm{mg} \cdot 1^{-1}$. The calibration equation was $A_{\mathrm{r}}=0.06739 C+0.00227(n=6), C$ is the concentration of puerarin in plasma, $A_{\mathrm{r}}$ is the ratio of peak-area of puerarin to that of hydroxybenzaldehyde. The correlation coefficient was 0.991 . The detection limit was about $100 \mathrm{ng} / \mathrm{ml}$. Precision data were obtained by repeated analysis of plasma samples with concentration of $0.0922,0.461,4.160 \mathrm{mg} \cdot 1^{-1}$, RSD $\%$ of within-day $(n=5)$ were $5.73,7.82,0.43$ respectively; RSD $\%$ of betweenday $(n=3)$ were $9.35,2.91,1.56$. The method recoveries were $101.5 \%$, $98.1 \%, 101.9 \%(n=5)$ respectively. The results showed that the method is accurate and precise for assay in vivo.

Data Analysis: The $A U C_{0} \infty$ was calculated by adding the area from time 0 to time $t\left(A U C_{0-t}\right)$ and the area from time $t$ to infinity $\left(A U C_{t-\infty}\right)$. The former was calculated using the trapezoidal formula and the latter by dividing the last measurable plasma drug concentration by the elimination rate constant $\left(k_{\mathrm{e}}\right)$. The $k_{\mathrm{e}}$ was estimated from the terminal slope of the individual plasma concentration-time curves after logarithmic transformation of the

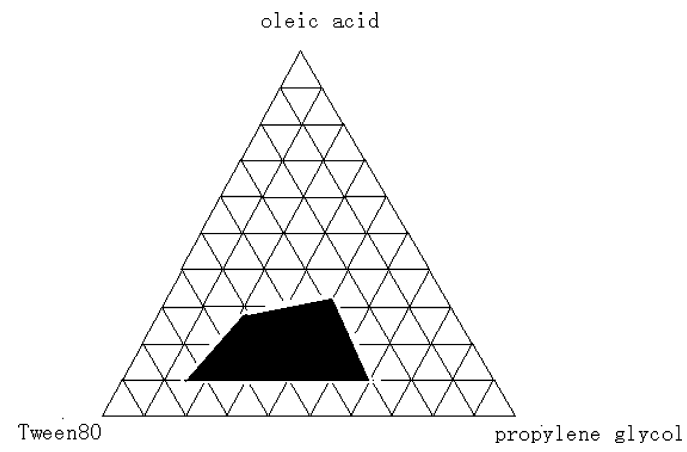

Fig. 1. Ternary Phase Diagram of Mixture Consisting of Oleic Acid, Propylene Glycol and Tween 80

The black domain indicate SEDDS region.

plasma concentration values and application of linear regression (calculated by program $3 \mathrm{p} 87$ which is the software to obtain pharmacokinetic parameters when input data of individual plasma concentration).

\section{Results and Discussion}

Solubility of Puerarin in Various Oils and Surfactants From the results of Table 2, among three oils of medium chain fatty acids (MCT), soybean oils (SO) and oleic acid (OA), puerarin appeared to have higher solubility in oleic acid. The possible reason might be that oleic acid had higher polarity than other two oils result in dissolving more isoflavone compound such as puerarin. Puerarin was stable in all three oils at $60^{\circ} \mathrm{C}$ for $10 \mathrm{~d}$. So oleic acid was selected for formulation development. Hydrophilic surfactants with HLB $>10$ are much superior at the providing fine, uniform emulsion droplets. Therefore two surfactants of Tween 80 and Cremophor RH40 were selected. Tween 80 provided a higher solubility of puerarin $(168.5 \mathrm{mg} / \mathrm{g}$ versus $103.3 \mathrm{mg} / \mathrm{g}$ ). For SEDDS formulations, the mixture should be a clear, monophasic liquid at ambient temperature and should have good solvent properties to allow presentation of the drug in solution. The dose of puerarin in the formulation was $100 \mathrm{mg}$, higher than $10 \%$, so propylene glycol as cosurfactant was added to improve solubility of puearin. The phase diagram was obtained (Fig. 1) in which the black domain indicate SEDDS region. From these studies, six SEDDS formulations (Table 2) with varying concentrations of oleic acids (16-40\%), Tween $80(13-55 \%)$, propylene glycol (17-40\%) were developed for further characterization.

Evaluation of Self-Emulsifying Performances Visual observations may provide important information about the self-emulsifying properties of the mixture and resulting dispersion system. Table 3 showed the results of mean droplet size and stability after centrifugation of emulsions of six SEDDS formulations. The SEDDS containing $0-20 \%$ (w/w) of Tween 80 did not form an emulsion, however, the SEDDS containing higher than $24 \%$ of Tween 80 rapidly formed an emulsion which appeared to be stable and no coalescence and no phase-separation were noted after centrifu- 
Table 2. Composition of Different SEDDS Formulations

\begin{tabular}{ccccc}
\hline \hline \multirow{2}{*}{$\begin{array}{c}\text { Formulation } \\
\text { No. }\end{array}$} & \multicolumn{4}{c}{ Composition/\%,w/w } \\
\cline { 2 - 5 } & Drug & Oil & Surfactant & Cosurfactant \\
\hline F1 & 11.6 & 16.7 & 53.8 & 17.9 \\
F2 & 13.3 & 22.2 & 43.0 & 21.5 \\
F3 & 13.5 & 17.5 & 34.5 & 34.5 \\
F4 & 19.3 & 20.7 & 24.0 & 36.0 \\
F5 & 23.3 & 31.1 & 15.2 & 30.4 \\
F6 & 20.4 & 26.3 & 13.3 & 40.0 \\
\hline
\end{tabular}

Table 3. The Results of Evaluation of Self-Emulsifying Performances

\begin{tabular}{ccl}
\hline \hline No. & Mean droplet size $(\mathrm{nm})$ & Results after centrifuge \\
\hline F1 & 216 & No separation \\
F2 & $210^{*}$ & No separation \\
F3 & $228^{*}$ & No separation \\
F4 & $248^{*}$ & No separation \\
F5 & $\mathrm{ND}^{a)}$ & Coalencense at surface \\
F6 & $\mathrm{ND}^{a)}$ & Phase separation \\
\hline
\end{tabular}

${ }^{a)} \mathrm{ND}$ : not determined, $p>0.05$ between $\mathrm{F} 1$ and F2, $* p<0.05$ between F2 and F3, F3 and F4.

gation. Formula 1 (F1) with $53.8 \%$ Tween 80 formed emulsion which the mean diameter was $216 \mathrm{~nm}$, Formula 2 (F2) with $43 \%$ Tween 80 formed emulsion which the mean diameter was $210 \mathrm{~nm}$, Formula 3 (F3) with $34.5 \%$ Tween 80 formed emulsion which the mean diameter was $228 \mathrm{~nm}$, Formula 4 (F4) with $24 \%$ Tween 80 formed emulsion which the mean diameter was $248 \mathrm{~nm}$. An analysis of variance procedure (one-way ANOVA) was used to compare the values of the formulations (F1, F2, F3, F4) with regard to the mean droplet sizes. It seemed that the mean diameter decreased with the amount of Tween 80 increased. But when the amount of Tween 80 increased to some extent, the mean diameter did not decrease further. A few studies have reported similar trends in droplet size with increases in surfactant concentration for various self-emulsifying systems ${ }^{13)}$ and explained that this phenomenon could be attributed to ejection of oil droplets into the aqueous phase result from the increased surfactant concentration. ${ }^{14)}$ On the other hand, it might be related to the addition of cosurfactant in the formulation. The ratio of cosurfactant/surfactant in the formulation F1, F2, F3, and F4 varied from $1: 3$ to $3: 2$. The minimum droplet size of resultant emulsion was found when the ratio of cosurfactant/surfactant was $1: 2$ in SEDDS. Many studies have reported that when a cosurfactant is added to the system, it further lowers the interfacial tension between the oil and water interface and also influences the interfacial film curvature, which thereby readily deforms around oil droplets. ${ }^{15)}$ In SEDDS formulation, the addition of surfactant as well as cosurfactant can both affect the properties of resulting emulsions.

Pharmacokinetics in Beagle Dogs The mean plasma concentration versus time profiles after administration of the oral SEDDS formulation (oleic acid 17.5\%, Tween $8034.5 \%$ and propylene glycol 34.5\%) and after i.v. administration of the injection $(100 \mathrm{mg} / \mathrm{ml}$ puerarin in $30 \%$ propylene glycol solution) were presented in Fig. 2. From the mean values of $A U C_{0-\infty}$ estimated from the individual plasma drug concen-

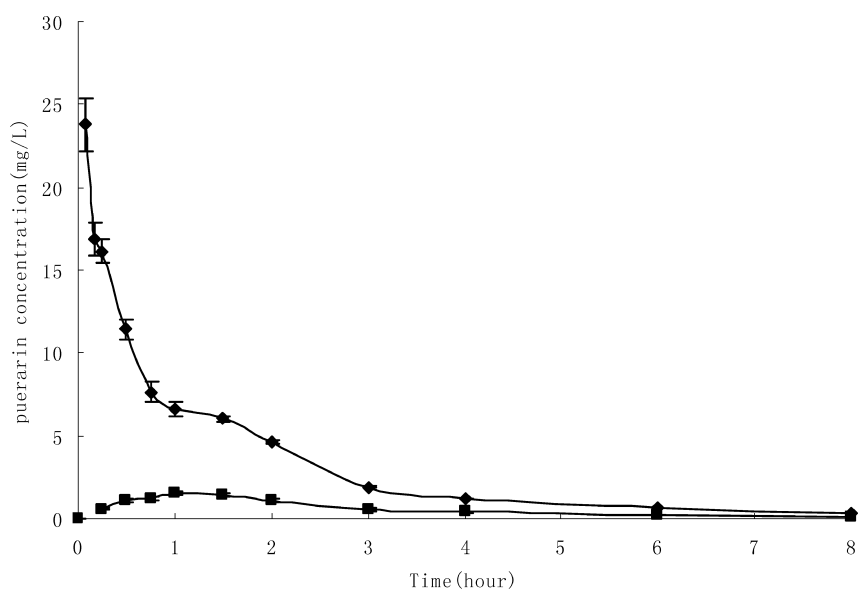

Fig. 2. Plasma Concentration-Time Curve of Puerarin in Beagle Dogs with Dose of $100 \mathrm{mg}(n=6)$

ם, SEDDS (F3) for p.o.; $\bullet$, injection for i.v.

tration profiles of puerarin, its absolute bioavailability was up to $24.8 \%$. Because no oral preparation was used in clinical, so puerarin suspension (suspended in $2 \% \mathrm{CMC}-\mathrm{Na}$ ) was prepared and administered to dog with puerarin dose of $100 \mathrm{mg}$. The plasma concentrations were assayed by the same method as described above. The results showed that after an oral administration of puerarin suspended in CMC-Na, parent compound in plasma could not be detected in all data points because they were all below the detected limit of the method $(100 \mathrm{ng} / \mathrm{ml})$. The peak plasma concentrations of puerarin with SEDDS were significantly higher than those after suspended in CMC-Na $(1.5 \mathrm{mg} / \mathrm{l}$ vs. $<0.1 \mathrm{mg} / \mathrm{l})$. From these results, it concluded that puerarin absorption can be improved with SEDDS formulation.

Recently the intestinal absorption and metabolism of isoflavones had been studied to better understand the mechanisms responsible for their low oral bioavailability. Extensive metabolism and enteric recycling in the intestine significantly contributes to its low bioavailability. ${ }^{16)}$ From these studies, there may be other possible reasons for enhanced uptake of puerarin formulated as SEDDS from the GI tract. In some cases, as shown recently, excipients incorporated in SEDDS/ self-microemulsifying drug delivery systems (SMEDDS) can inhibit both presystemic drug metabolism and intestinal efflux mediated by P-gp resulting in an increased oral absorption of drugs (Chervinsky D. S., et al.). However, it should be noticed that the bioavailability of puerarin in SEDDS formulation is still low. In further study, SEDDS co-administered with P-gp inhibitors, D- $\alpha$-tocopheryl polyethylene glycol 1000 succinate (TPGS) and Cremophor in the formulation can be designed and developed.

The pharmacokinetic behaviors of SEDDS formulations (F2, F3, F4) were investigated in beagle dogs. The bioavailability of three different SEDDS formulations were compared using the pharmacokinetic parameters, peak plasma concentration $\left(C_{\max }\right)$, time to reach peak plasma concentration $\left(T_{\max }\right)$ and total area under the plasma concentrationtime curve $\left(A U C_{0-\infty}\right)$. The mean plasma concentration versus time profiles of three SEDDS formulations were shown in Fig. 3. There was a statistically significant difference among the $A U C_{0-\infty}(p<0.01)$, as well as the $C_{\max }(p<0.01)$ values of the three preparations. Further analysis of the data using 


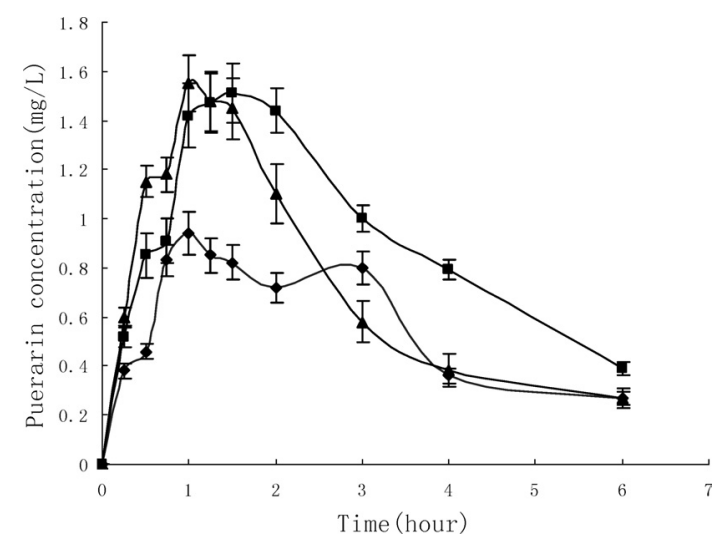

Fig. 3. Plasma Drug Concentration-Time Curve of Different Puerarin SEDDS in Beagle Dogs $(n=6)$

$\mathbf{\square}, \mathrm{F} 2 ; \boldsymbol{\Delta}, \mathrm{F} 3 ; \bullet, \mathrm{F} 4$.

Tukey's test showed a statistically significant difference between F2 and F4 $(p<0.01)$ as well as F3 and F4 $(p<0.01)$ with regard to the values of $A U C_{0-\infty}$ and $C_{\text {max }}$, but not between those of F2 and F3 $(p>0.05)$. So F3 with oleic acid $(17.5 \%)$, Tween $80(34.5 \%)$ and propylene glycol $(34.5 \%)$ was chosen as the optimized SEDDS formulation of puerarin and its absolute bioavailability was investigated further.

\section{Conclusion}

The present study demonstrates that self-emulsifying drug delivery system (SEDDS) could be a potential way of puer- arin administered orally.

Acknowledgements We would like to express our gratitude to Prof. Jinxiu R. for consultation in plasma concentration testing. This work was supported by National Medical foundation of PLA in China.

\section{References}

1) Robinson J. R., Bull. Technique Gattefosse, 89, 11-13 (1996).

2) Aungst B. J., J. Pharm. Sci., 82, 979-987 (1993).

3) Gershanik T., Benita S., Eur. J. Pharm. Biopharm., 50, 179-188 (2000).

4) Khoo S. M., Humberstone A. J., Charman W. N., Int. J. Pharm., 167, $155-164$ (1998).

5) Kim J. Y., Ku Y. S., Int. J. Pharm., 194, 81-89 (2000).

6) Kommuru T. R., Gurley B., Khan M. A., Reddy I. K., Int. J. Pharm., 212, 233-246 (2001).

7) Nazzal S., Smalyukh I. I., Lavrentovich O. D., Khan M. A., Int. J. Pharm., 235, 247-265 (2002).

8) Gursoy R. N., Benita S., Biomed. Pharmacother., 58, 173-182 (2004).

9) Chervinsky D. S., Brecher B. L., Hoelcle M. J., Anticancer Res., 13 93-96 (1993).

10) Li Y., Pan W.-S., Chen S.-L., Yang D.-J., Chen S.-C., Xu H.-X., J. Chin. Pham., 41, 1162-1167 (2006).

11) Craig D. Q. M., Bull. Technique Gattefosse, 86, 21-31 (1993).

12) Zhang Z. R., You X. J., He Q., Liao G.-T., J. Chin. Pham., 32, 104 106 (1997).

13) Craig D. Q. M., Barker S. A., Banning D., Booth S. W., Int. J. Pharm., 114, 103-110 (1995).

14) Pouton C. W., Adv. Drug Del. Rev., 25, 47-58 (1997).

15) Gao P., Witt M. J., Haskell R. J., Zamora K. M., Shifflett J. R., Pharm. Dev. Technol., 9, 301-309 (2004).

16) Chen J., Lin H., Hu M., J. Pharmacol. Exp. Ther., 304, 1228-1235 (2003). 